\title{
Facile synthesis of $(\mathrm{CdZn}) \mathrm{Se}$ nanocrystalline thin films via arrested precipitation technique (APT) for photovoltaic application
}

\author{
Chaitali. S. Bagade, Vishvnath. B. Ghanwat, Kishorkumar. V. Khot, Pallavi. B. Patil, \\ Rahul. M. Mane, P. N. Bhosale* \\ Materials Research Laboratory, Department of Chemistry, Shivaji University, Kolhapur 416004, India \\ *p_n_bhosale@rediffmail.com
}

PACS 81

DOI 10.17586/2220-8054-2016-7-3-553-557

\begin{abstract}
Nanocrystalline $(\mathrm{Cd} Z n)$ Se thin films have been successfully synthesized via a simple and cost effective arrested precipitation technique. The deposition and synthetic strategy of $(\mathrm{CdZn}) \mathrm{Se}$ thin films exert appreciable influence on the photovoltaic properties of solar cells. In this paper, systematic characterizations of optostructural, morphological, compositional and electrochemical properties have been carried out. The optical band gap was evaluated from UV-Vis-NIR spectra at wavelengths ranging from $400-1100 \mathrm{~nm}$. X-ray diffraction (XRD) pattern reveals that the deposited film was nanocrystalline in nature and exhibited a cubic crystal structure. The dependency of microstructural parameters such as crystallite size has been studied. Scanning electron microscopy (SEM) images demonstrate that surface morphology was uniform, dense, smooth and well adhered to substrate surface. The as-deposited nanorystalline $(\mathrm{Cd} Z \mathrm{Zn}) \mathrm{Se}$ thin film exhibits $0.61 \%$ conversion efficiency at room temperature.
\end{abstract}

Keywords: $(\mathrm{CdZn}) \mathrm{Se}$, arrested precipitation technique (APT), thin films, semiconductor material, solar cells.

Received: 2 February 2016

Revised: 11 April 2016

\section{Introduction}

In recent years, the field of nanocrystalline semiconducting thin films is rapidly expanding. The increasing interest for these materials is due to the fact that these are characterized by properties which are substantially different from the corresponding ones for bulk semiconductors [1]. In this regard, group II-VI semiconductors are considered important technological materials due to their potential applications in optoelectronic devices [2]. Among these, cadmium zinc selenide $(\mathrm{CdZn})$ Se is found to be an excellent material with a band gap value around $1.9 \mathrm{eV}$ which makes it fairly interesting for the fabrication of solar cells through the photoelectrochemical route [3]. $(\mathrm{CdZn}) \mathrm{Se}$ is a promising ternary material because of its tunable parameters, such as band gap and surface morphology. The most important applications of $(\mathrm{CdZn}) \mathrm{Se}$ thin film is in solar cells, high efficiency thin film transistors, light emitting diodes, laser diodes and electroluminescent devices [4-7].Various techniques have been used for the synthesis of $(\mathrm{Cd} Z n)$ Se thin films such as electrodeposition, chemical bath deposition (CBD), screen printing followed by sintering and metal organic chemical vapor deposition (MOCVD) [8-10]. One of the disadvantages of this technique is that some of them need sophisticated instrumentation along with vacuum and high temperature which increases the production cost of the material. However, the solution based deposition method, i.e. arrested precipitation technique offers the possibility of depositing thin films at low temperature under atmospheric conditions and at low fabrication cost. As a one step, environment friendly and low energy consumption aqueous technique, APT is based on controlled release of metal ions from metal complexes and reaction with chalcogen ions accordingly Ostwald ripening law [11].

\section{Experimental}

\subsection{Deposition of thin film}

We have developed a simple, rapid, low cost and environmentally friendly chemical method using nontoxic reagents for the deposition of proposed thin films because of its various advantages such as, large area deposition, relatively low temperature processes, no restriction on the use of substrate, reproducibility and most significantly no requirement of sophisticated equipment. A modified chemical deposition technique which is combination of CBD and controlled chemical growth process (CCGP) is employed. This modified technique is known as arrested precipitation technique (APT). It is based on the controlled release of metal and chalcogen ions and characterized by simple formulation, ease of set up and has the potential to replace 
expensive energy and equipment demanding techniques. Additionally, surface morphology can be tuned by adjusting the $\mathrm{pH}$ of the bath solution, deposition time, temperature and the reagent concentration.

In a typical synthesis, $8 \mathrm{ml}$ of $0.05 \mathrm{M}$ [Cd-EDTA] and $12 \mathrm{ml}$ of $0.2 \mathrm{M}$ [Zn-EDTA] solution was taken in reaction beaker. The $\mathrm{pH}$ of bath was adjusted to 10.4 by dropwise addition of ammonia. Next, $20 \mathrm{ml}$ of $0.25 \mathrm{M} \mathrm{Na}_{2} \mathrm{SeSO}_{3}$ solution was added to reaction bath with constant stirring. The total volume of reaction bath was adjusted to $50 \mathrm{ml}$ by adding double distilled water then reaction mixture was stirred for 5 min to get homogeneous solution. Precleaned ITO glass substrates were immersed vertically close to the inner wall of reaction container and then kept at $50{ }^{\circ} \mathrm{C}$ temperature with constant substrate rotation $45 \mathrm{rpm}$ and deposition time was kept $4 \mathrm{~h}$. Various preparative parameters like $\mathrm{pH}$, temperature, concentration and time were finalized at initial stage of deposition After deposition, substrates were withdrawn from bath, sufficiently rinsed with double distilled water and dried at room temperature. The deposited thin film was uniform, peach in color and well-adhered to the glass substrate. This deposited thin film was used for subsequent investigations.

\subsection{Characterizations of thin film}

The thickness of the deposited thin films was measured by surface profiler (AMBIOS XP-1). A UV-Vis Spectrophotometer (Model: Shimadzu UV-1800) was used to record absorption spectra of deposited thin films in from $400-1100 \mathrm{~nm}$. The structural properties of the thin films were studied using X-ray Diffractometer (Bruker AXS, D8 Model) using $\mathrm{Cu} \mathrm{K} \alpha(\lambda=1.5418 \AA)$ radiation for $2 \theta$ ranging from $10^{\circ}$ to $80^{\circ}$. Surface morphology and elemental analysis were examined using Scanning Electron Microscope (SEM) equipped with Energy Dispersive Spectroscopic (EDS) analyzer (JEOL-JSM-6360A). Photoelectrochemical (PEC) measurement was carried out by using $(\mathrm{CdZn})$ Se thin film as photoanode in dark and under illumination using a $500 \mathrm{~W}$ tungsten filament lamp (intensity $30 \mathrm{mWcm}^{-2}$ ) at electrochemical workstation (AUTOLAB PGSTAT 100 potentiostat). The photoelectrochemical cell was a two electrode system: (CdZn)Se film deposited on ITO substrate is a working electrode with active surface area of $1 \mathrm{~cm}^{2}$ and graphite as counter electrode in sulphide/polysulphide as redox electrolyte.

\section{Results and discussion}

\subsection{Thickness measurement}

The thickness of the deposited thin film was found to be $730 \mathrm{~nm}$. Thickness of the deposited films is controlled by two independent variables such as uniform growth and surface morphology. The process of precipitation of a substance in the solution depends on degree of supersaturation and formation of nucleus and its growth onto the substrate's surface.

\subsection{Optical and structural analysis}

Optical band gap energy of $(\mathrm{CdZn})$ Se thin film was calculated by using the optical absorption spectra. The optical absorption spectra of deposited (CdZn)Se thin film was recorded from 400-1100 nm, as shown in Fig. 1(a). Electronic transition between valence and conduction bands starts at the absorption edge, corresponding to the minimum energy difference between the lowest energy of conduction band and the highest energy of valence band in material. Fig. 1(a) clearly shows that maximum optical absorption is observed at around $650-750 \mathrm{~nm}$.

The band gap of $(\mathrm{CdZn})$ Se thin film was determined by extrapolating straight line to the energy axis using equation (1)

$$
\alpha=\frac{A(h \nu-E g)^{n}}{h \nu}
$$

where ' $\alpha$ ' is absorption coefficient, ' $h \nu$ ' is photon energy ' $A$ ' is a parameter that depends on the transition probability, ' $h$ ' is planck's constant, ' $E g$ ' is optical band gap energy of the material and exponent ' $n$ ' depends on nature of transition during absorption process. The value of $n$ is $1 / 2,3 / 2,2,3$ for direct allowed, direct forbidden, indirect allowed and indirect forbidden transitions, respectively. The nature of the plots suggest a direct and allowed type of transition, since linear dependence is obtained at $n=1 / 2$. The optical band gap value of $(\mathrm{CdZn})$ Se thin film $1.91 \mathrm{eV}$ was observed as shown in inset Fig. 1(a).

XRD pattern of $(\mathrm{CdZn})$ Se thin film was carried out at room temperature in the range of $10-80^{\circ}$ and shown Fig. 1(b). Broad and intense peaks in the XRD patterns confirm the nanocrystalline nature of thin film. XRD patterns of thin film exhibit peaks at $25.39^{\circ}, 42.17^{\circ}, 49.74^{\circ}$ and $60.85^{\circ}$ which are indexed to the (111), (220), (311) and (400) planes respectively of cubic CdSe (JCPDS card no. 19-0191). Also, the peaks at $12.47^{\circ}, 14.30^{\circ}, 20.13^{\circ}, 23.62^{\circ}, 29.32^{\circ}$ and $31.83^{\circ}$ are indexed to the (111), (200), (220), (311), (400) and (331) planes of cubic ZnSe (JCPDS card no. 02-0479). The XRD pattern of (CdZn)Se 


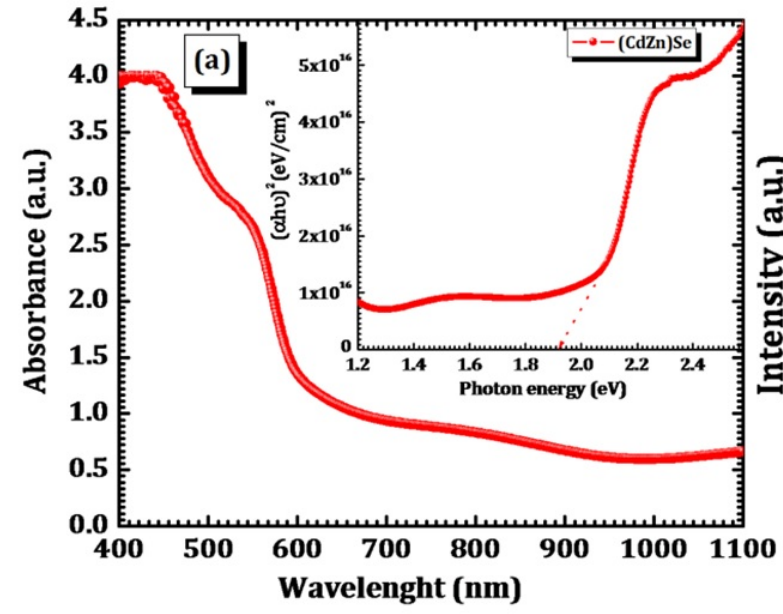

a

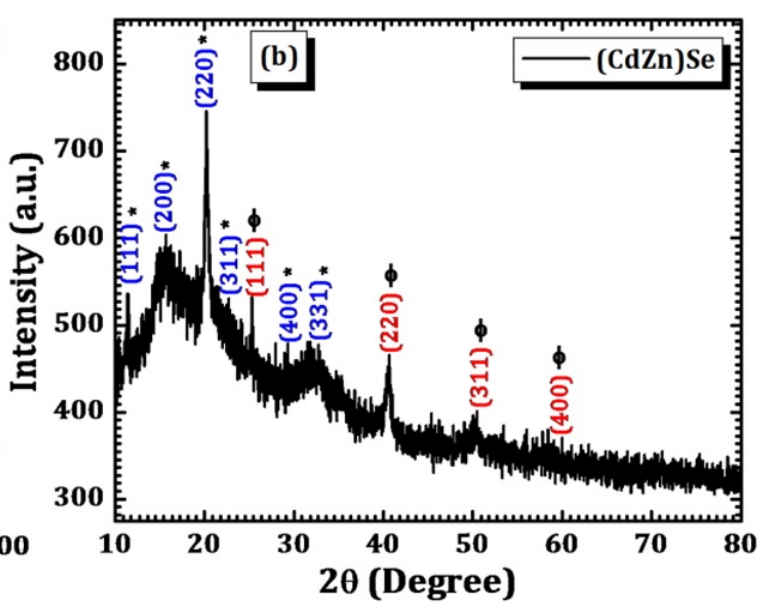

b

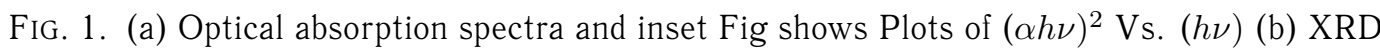
pattern of $(\mathrm{CdZn})$ Se thin film

nanocrystals was located between those for CdSe and ZnSe materials. Such phase formation of CdSe and $\mathrm{ZnSe}$ provides strong evidence for $(\mathrm{Cd} \mathrm{Zn})$ Se solid solution formation, which was in good agreement with the reported value $[12,13]$. The average crystallite size was calculated from XRD patterns using Debye Scherrer's eq. (2)

$$
D=\frac{0.94 \lambda}{\beta \cos \theta},
$$

where ' $\lambda$ ' is used X-ray wavelength (1.5406 $\AA$ ), ' $\beta$ ' is full width of half-maximum (FWHM) in radians and ' $\theta$ ' is Bragg's angle. The calculated crystallite size was $24 \mathrm{~nm}$ for the $(\mathrm{CdZn})$ Se thin film.

\subsection{Morphology and compositional analysis}

In order to study the microstructures of $(\mathrm{CdZn}) \mathrm{Se}$ thin films, scanning electron microscopy (SEM) was used. Fig. 2 shows high and low magnification SEM micro images of $(\mathrm{CdZn})$ Se thin films. It shows formation of densely packed nanospheres like smooth homogenous surface morphology which was dispersed uniformly on the substrate surface. The presence of elements in deposited thin film was confirmed by EDS technique. Fig. 2(e) shows elemental distribution of the constituent elements for typical (CdZn)Se thin film. The elemental analysis was carried out only for $\mathrm{Cd}, \mathrm{Zn}$, and Se elements. The peaks at $3.55,1.10$ and $1.70 \mathrm{keV}$ confirm the presence of $\mathrm{Cd}, \mathrm{Zn}$ and Se in thin film, respectively [14].

\subsection{Photoelectrochemical performance}

The PEC performance of $(\mathrm{CdZn})$ Se thin film was verified using a standard two electrode configuration in the dark and under an illumination of $30 \mathrm{~mW} / \mathrm{cm}^{2}$ light intensity and in $0.5 \mathrm{M}$ sulfide/polysulfide redox electrolyte. After illumination, shifting of $\mathrm{J}-\mathrm{V}$ curve in the fourth quadrant suggests that electrons were generated due to illumination, the magnitude of open circuit voltage increases with negative polarity towards $(\mathrm{CdZn})$ Se electrode, indicating cathodic behavior of the photovoltage, which confirms that $(\mathrm{CdZn}) \mathrm{Se}$ thin films are n-type. The current voltage $(\mathrm{J}-\mathrm{V})$ characteristics of glass/ITO/ (CdZn)Se $0.5 \mathrm{M}$ polysulfide/graphite were measured. From the J-V measurements, the obtained value of Jsc was $1.162 \mathrm{~mA} / \mathrm{cm}^{2}$ and corresponding values of Voc was $581 \mathrm{mV}$, respectively. Closely packed nanospheres improve the carrier transport mechanism and minimize the surface trap states. Such types of interconnected nanospheres provide higher effective surface area for light absorption. A conversion efficiency of $0.61 \%$ was achieved for $(\mathrm{Cd} Z \mathrm{n}) \mathrm{Se}$ thin film [15].

\section{Conclusion}

In conclusion, we report a facile chemical route for the deposition of $(\mathrm{Cd} \mathrm{Zn}) \mathrm{Se}$ thin film by using a simple and cost effective APT. From optical measurements, we can conclude that optical band gap was the direct allowed type, having a band gap energy $1.91 \mathrm{eV}$. X-ray diffraction pattern illustrated cubic crystal structure 


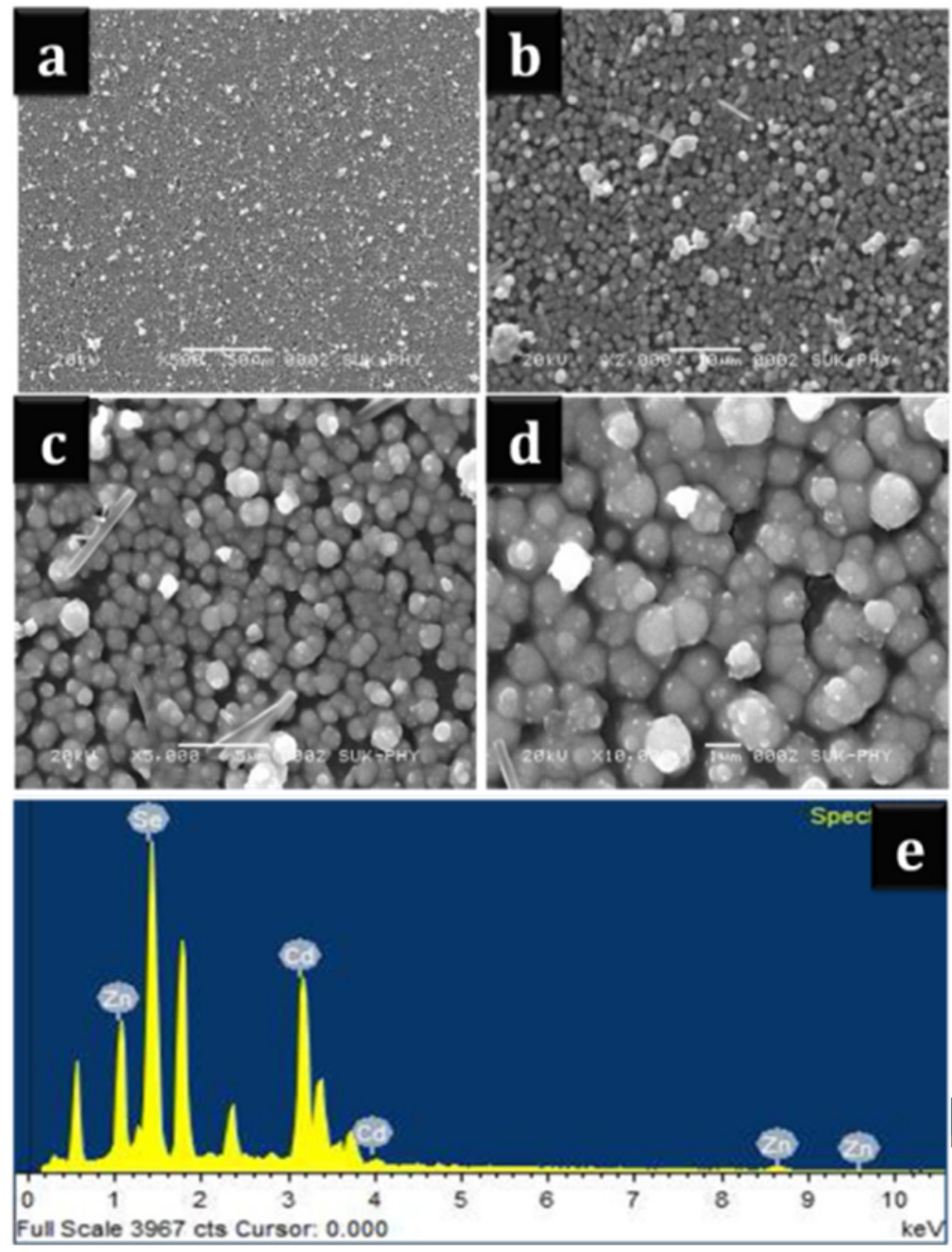

FIG. 2. (a-d) Low and high magnification SEM images and (e) EDS spectra of (CdZn)Se thin film

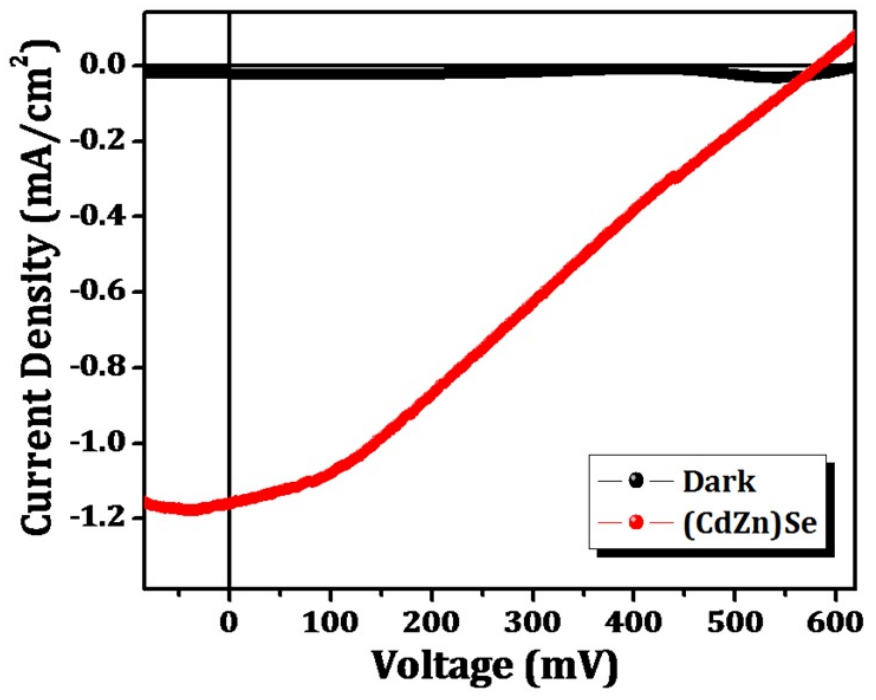

FIG. 3. $J-V$ characteristics of $(\mathrm{CdZn}) \mathrm{Se}$ thin film in sulphide/polysulphide electrolyte 
for both CdSe and $\mathrm{ZnSe}$ phases. SEM study illustrated that nanosphere like morphology for (CdZn)Se thin film was observed. Such morphology shows high efficiency due to large surface area for the absorption of light. EDS analysis shows presence of all elements ( $\mathrm{Cd}, \mathrm{Zn}$ and $\mathrm{Se}$ ) in stoichiometric form. The highest conversion efficiency was obtained for $(\mathrm{CdZn})$ Se thin film $(0.61 \%)$ thin film. Overall achieved results revealed that newly devised APT is a suitable method for the synthesis of different metal chalcogenide thin films.

\section{Acknowledgement}

One of the authors, Chaitali S. Bagade is very much thankful to Department of Science and Technology (DST), New Delhi for providing DST-INSPIRE fellowship for financial support (Registration No. IF 140571). This work is also supported by the Priority Research Centre Program through the National Research Foundation of Korea (NRF) funded by the Ministry of Education, Science and Technology (2009-0094055).

\section{References}

[1] Ubale U. Effect of complexing agent on growth process and properties of nanostructured $\mathrm{Bi}_{2} \mathrm{~S}_{3}$ thin films deposited by chemical bath deposition method. Mater. Chem. \& Phys., 2010, 121, P. 555-560.

[2] Bagade C. S., Ghanwat V. B., Khot K. V., Bhosale P. N. Efficient improvement of photoelectrochemical performance of CdSe thin film deposited via arrested precipitation technique. Mate. Lett., 2016,164, P. 52-55.

[3] Zhai R., Wang S., Xu H. Y., Wang H., Yan H. Rapid formation of CdS, ZnS thin films by microwave-assisted chemical bath deposition. Mater. Lett., 2005, 59, P. 1497-1501.

[4] Wu X., Yu Y., Liu Y., Xu Y., Liu C., Zhang B. Synthesis of Hollow $\mathrm{Cd}_{x} \mathrm{Zn}_{1-x}$ Se Nanoframes through the Selective Cation Exchange of Inorganic-Organic Hybrid ZnSe-Amine Nanoflakes with Cadmium Ions. Angew. Chem. Int. Ed., 2012, 51, P. 32113215.

[5] Bagade C.S., Mali S.S., Ghanwat V.B., Khot K.V., Patil P.B., Kharade S.D., Mane R.M., Desai N.D., Hong C.K., Patil P.S., Bhosale P.N. A facile and low cost strategy to synthesize $\mathrm{Cd}_{1_{-} x} \mathrm{Zn}_{x}$ Se thin films for photoelectrochemical performance: effect of zinc content. RSC Adv., 2015, 5, P. 55658-55668.

[6] Akaltun Y., Yıldırım M.A., Atesn A., Yıldırım M. Zinc concentration effect on structural, optical and electrical properties of $\mathrm{Cd}_{1-x} \mathrm{Zn}_{x}$ Se thin films. Mate. Res. Bull., 2012, 47, P. 3390-3396.

[7] Camargo-Gambo G.J., L. Pacheco J.S., Leon J.M. de, Conradson S.D., Hernańdez-Caldero I. Local structural characterization of Zn:Cd:Se ternary semiconductors. Thin Solid Films, 2005, 490, P. 165-167.

[8] Chavhan S.D., Mane R.S., Ganesh T., Lee W., Han S.H., Senthilarasu S., Lee S. H. Structural and optical properties of electrodeposited $\mathrm{Cd}_{0.7} \mathrm{Zn}_{0.3}$ Se thin films: Effect of annealing. J. Alloys Compd., 2009, 474, P. 210-213.

[9] Sutrave D.S., Shahane G.S., Patil V.B, Deshmukh L.P. Micro-crystallographic and optical studies on $\mathrm{Cd}_{1-x} \mathrm{Zn}_{x} \mathrm{Se}_{\mathrm{f}} \mathrm{hin}$ films. Mater. Chem. Phys., 2000, 65, P. 298-305.

[10] Shan C.X., Fan X.W., Zhang J.Y., Zhang Z.Z., Wang X.H., Lu Y.M., Liu Y.C., Shen D.Z., Lu S.Z. Fabrication of ZnCdSe quantum dots under Stranski-Krastanow mode, J. Cryst. Growth, 2004, 265, P. 541-547.

[11] Mezrag F., Mohamed W.K., Bouariss N. The effect of zinc concentration upon optical and dielectric properties of $\mathrm{Cd}_{1-x} \mathrm{Zn}_{x} \mathrm{Se}$. Phys. B, 2010, 405, P. 2272-2276.

[12] Cao J., Xue B., Li H., Deng D., Gu Y. Facile synthesis of high-quality water-soluble N-acetyl-1-cysteine-capped $\mathrm{Zn}_{1-x} \mathrm{Cd}_{x} \mathrm{Se}_{\mathrm{Zn}} \mathrm{ZnS}$ core/shell quantum dots emitting in the violet-green spectral range. J. Colloid Interface Sci., 2010, 34(8), P. 369-376.

[13] Sheng Y., Weia J., Liu B., Peng L. A facile route to synthesize CdZnSe core-shell-like alloyed quantum dots via cation exchange reaction in aqueous system. Mate. Res. Bull., 2014, 57, P. 67-71.

[14] Zhong X., Zhang Z., Liu S., Han M., Knoll W. Embryonic Nuclei-Induced Alloying Process for the Reproducible Synthesis of Blue-Emitting $\mathrm{Zn}_{x} \mathrm{Cd}_{1-x}$ Se Nanocrystals with Long-Time Thermal Stability in Size Distribution and Emission Wavelength. $J$. Phys. Chem. B., 2004, 108, P. 15552-15559.

[15] Kharade S.D., Pawar N.B., Mali S.S., Hong C.K., Patil P.S., Gang M.G., Kim J., Bhosale P.N. Effect of copper content on optostructural, morphological and photoelectrochemical properties of $\mathrm{MoBi}_{2-x} \mathrm{Cu}_{x} \mathrm{Se}_{4}$ thin films. J. Mater Sci, 2013, 48, P. 73007311. 